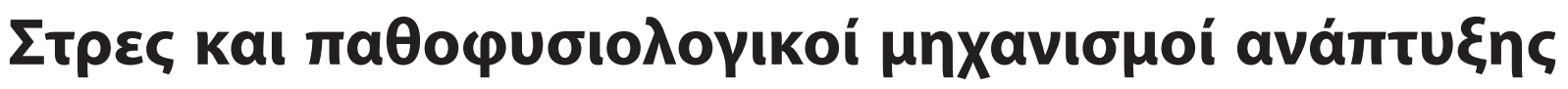

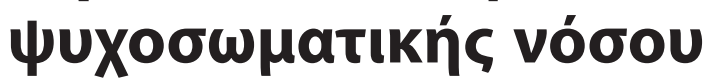

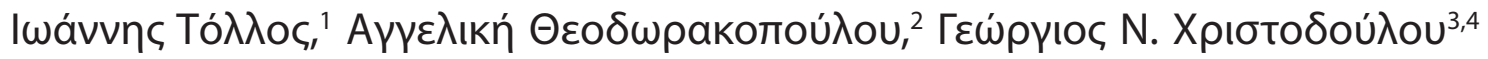

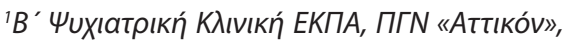

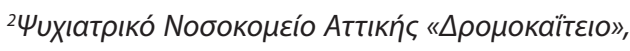

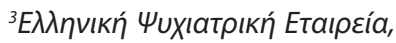

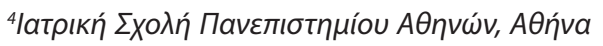

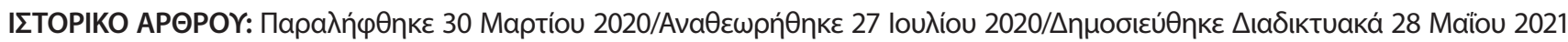

\begin{abstract}
ПЕРІАНЧН

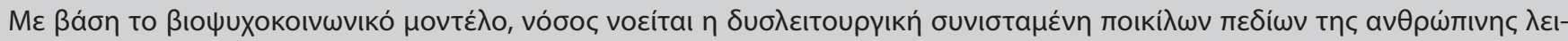

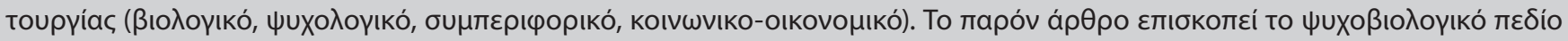

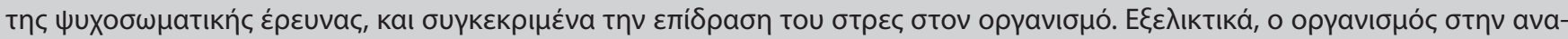

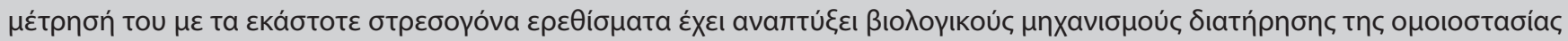

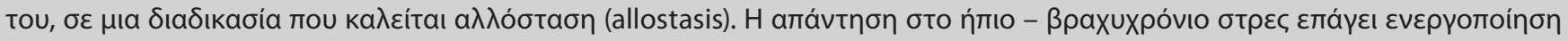

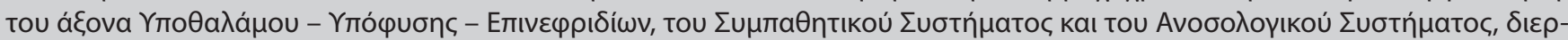

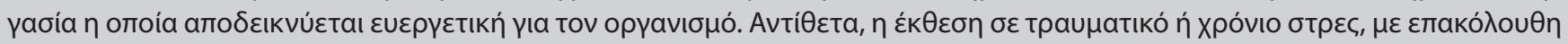

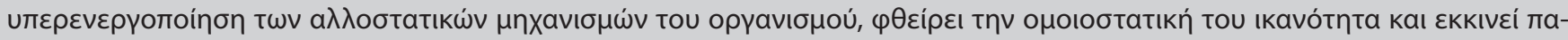

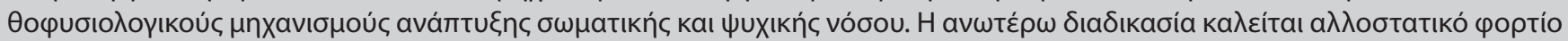

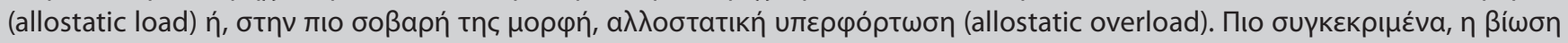

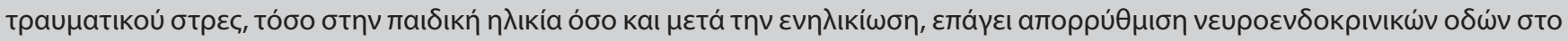

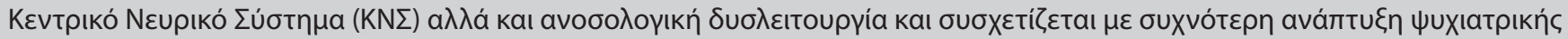

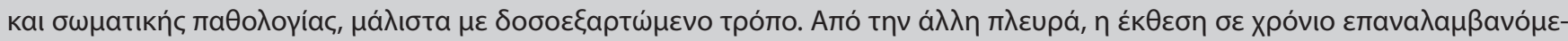

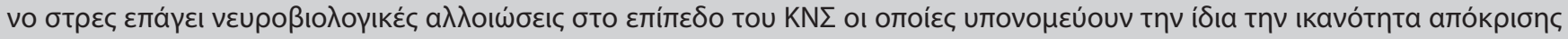

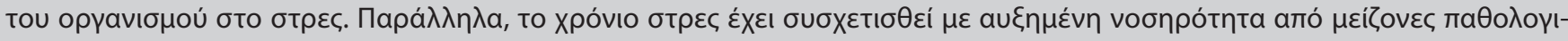

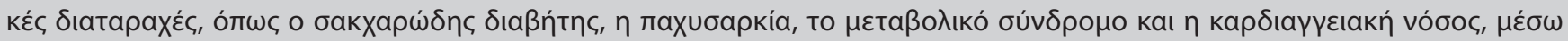

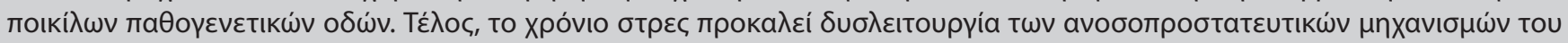

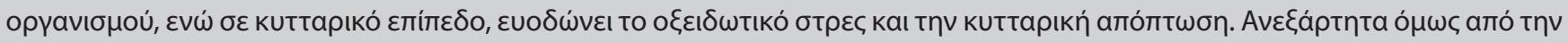

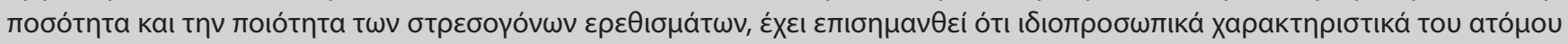

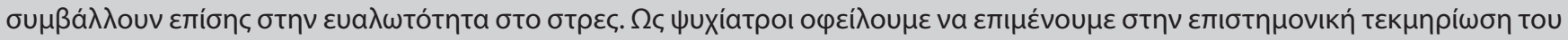

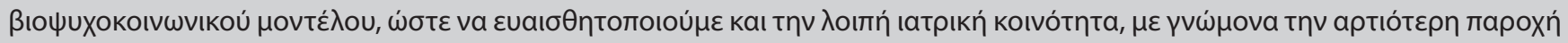

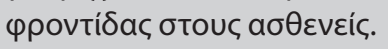

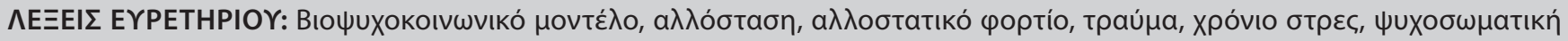
Vóooৎ

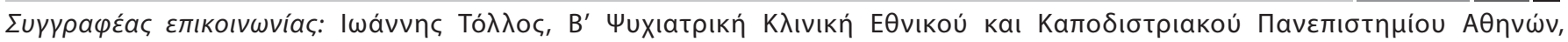

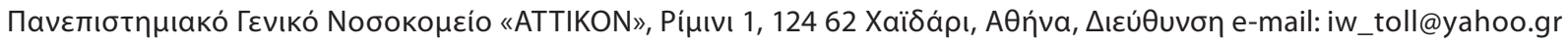




\section{Eıoapwpń}

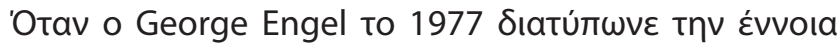

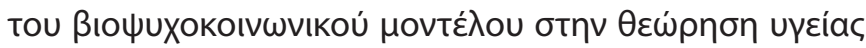

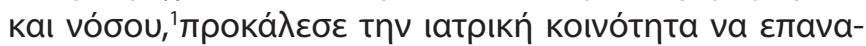

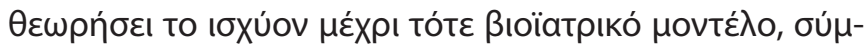

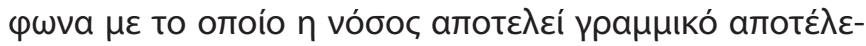

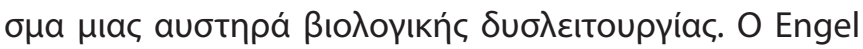

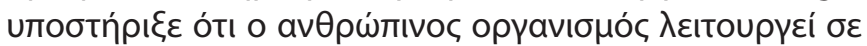

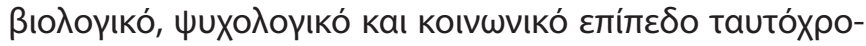

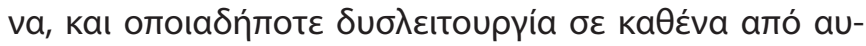

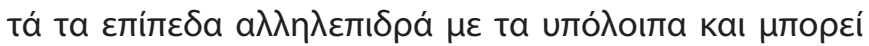

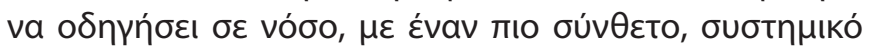

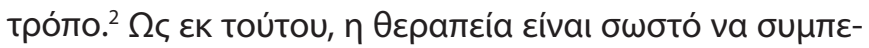

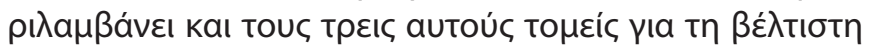

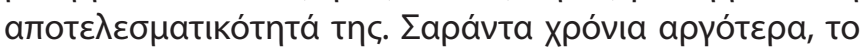

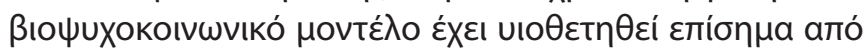

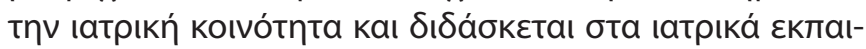

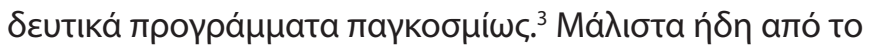

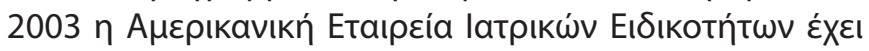

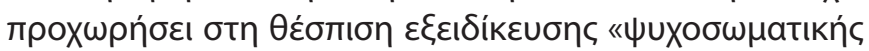

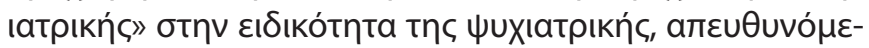

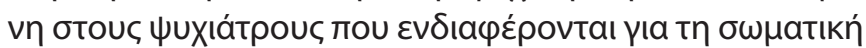
vóoo. ${ }^{2}$

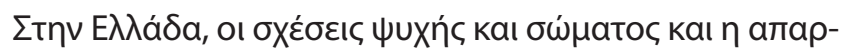

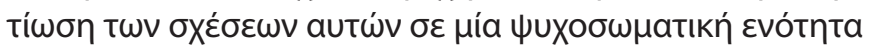

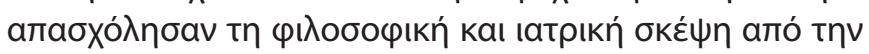

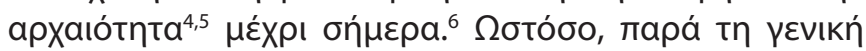

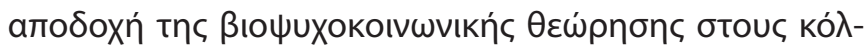

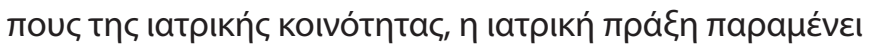

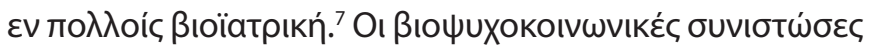

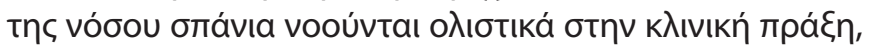

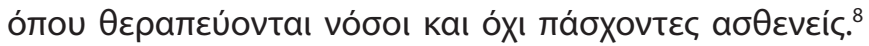

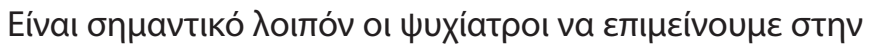

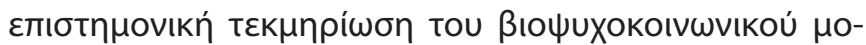

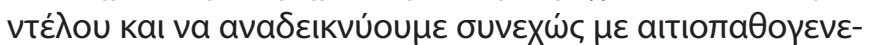

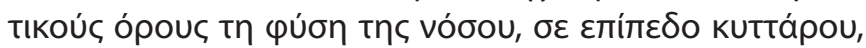

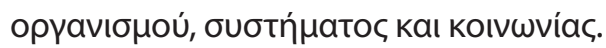

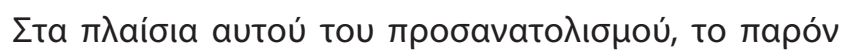

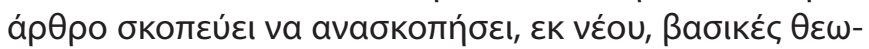

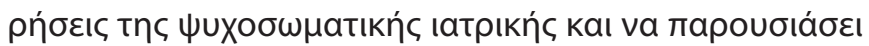

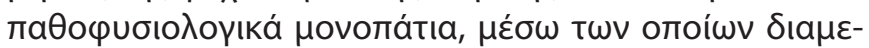

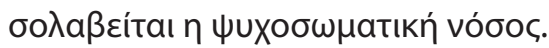

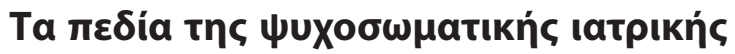

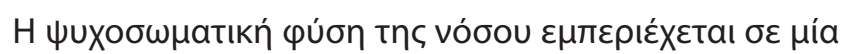

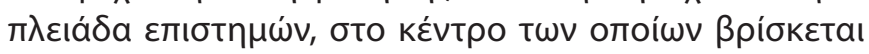

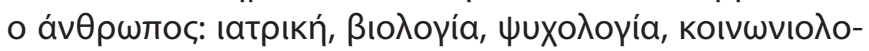

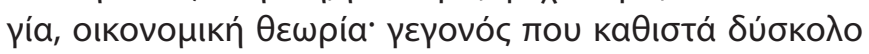

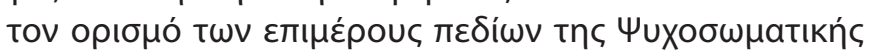

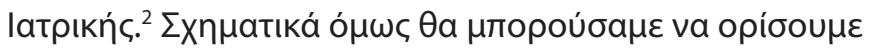

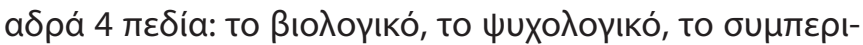

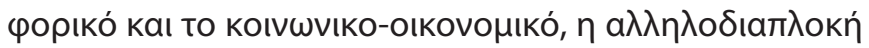

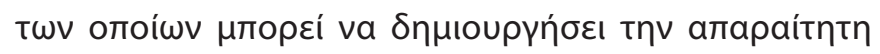

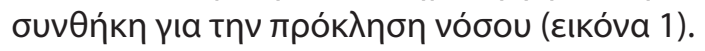

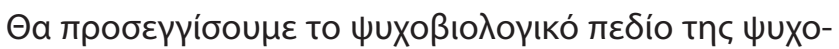

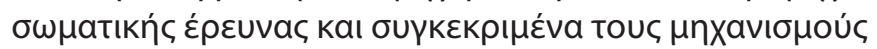

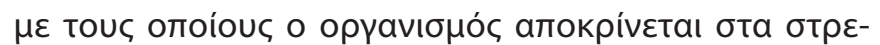

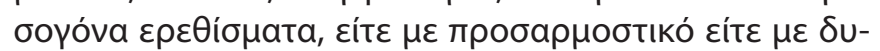
бтробарнобтіко́ тро́то.

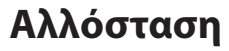

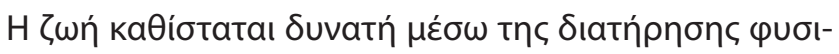

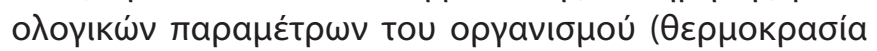

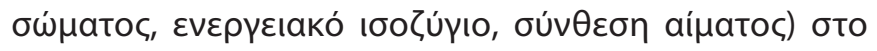

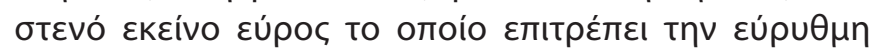

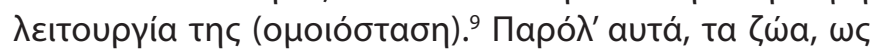

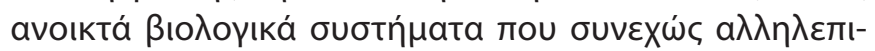

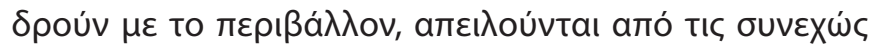

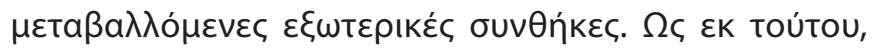

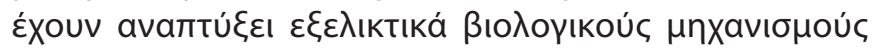

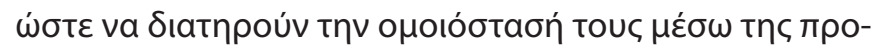

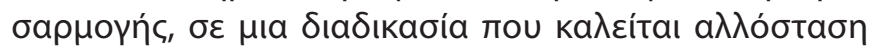

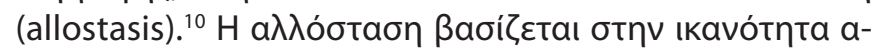

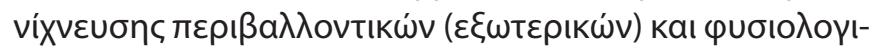

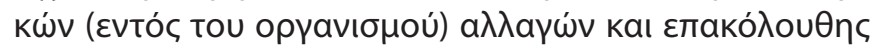

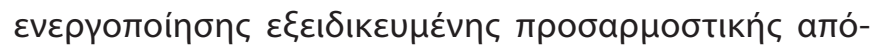
кpıonc.

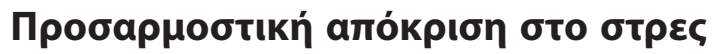

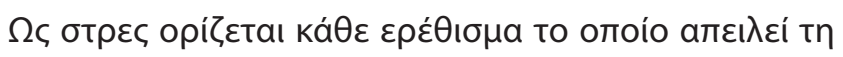

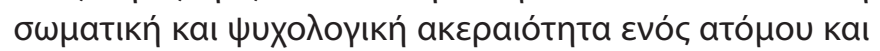

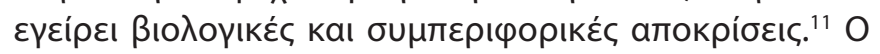

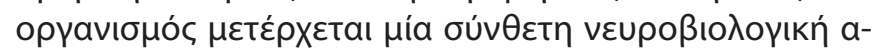

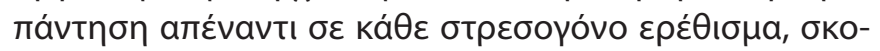

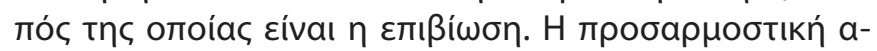

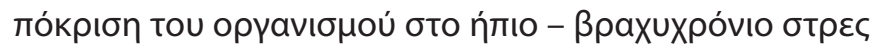

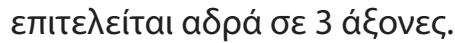

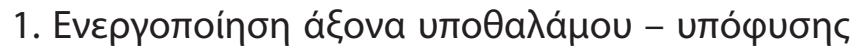

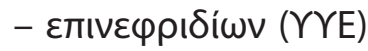

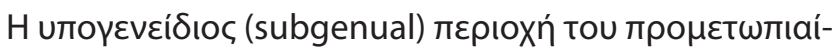

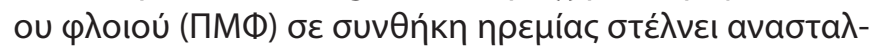

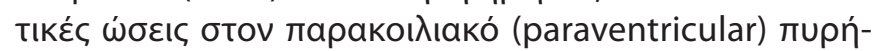

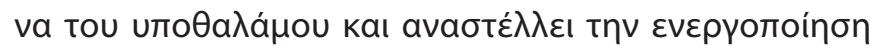

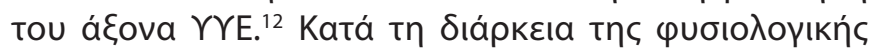

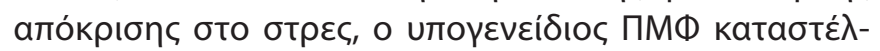

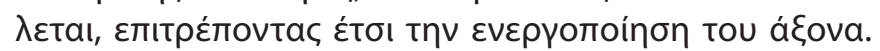

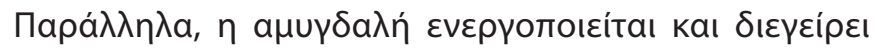

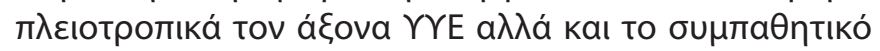

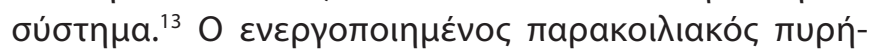

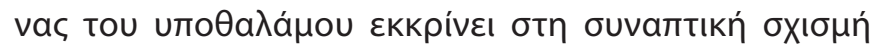

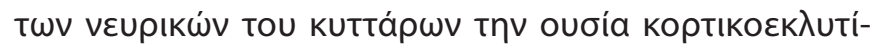

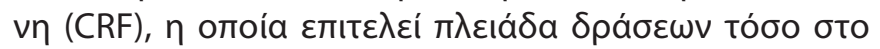

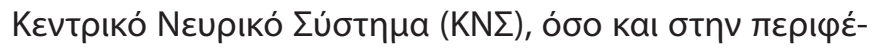




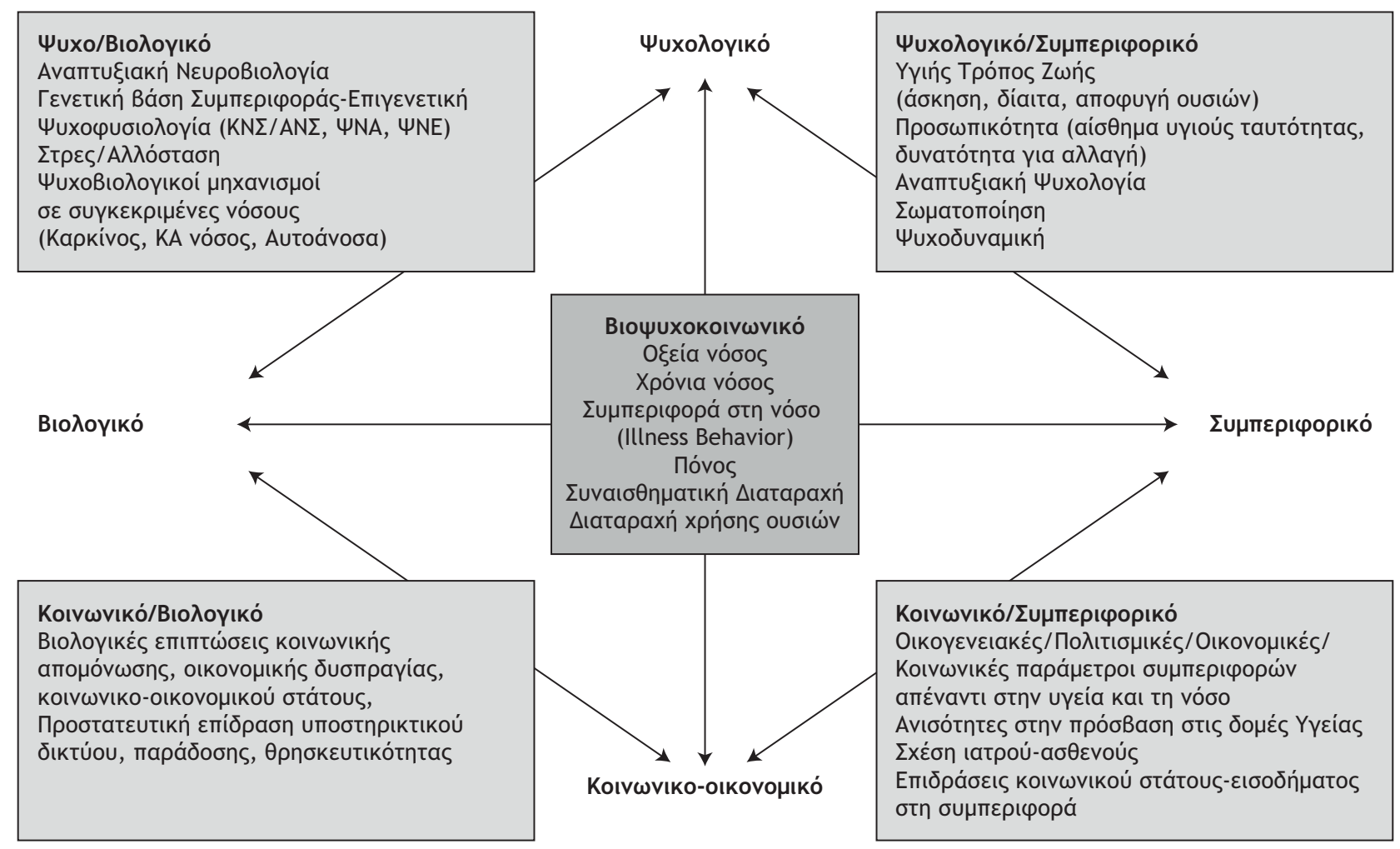

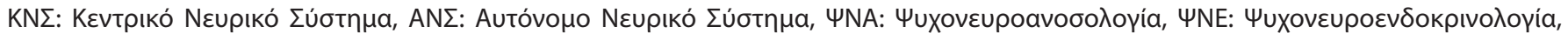

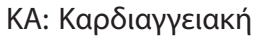

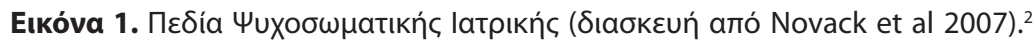

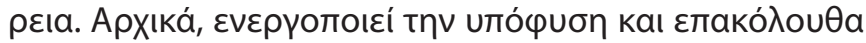

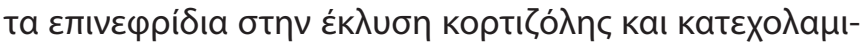

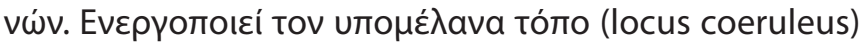

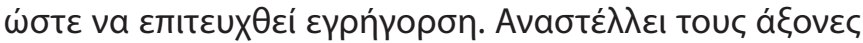

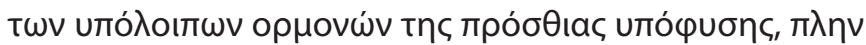

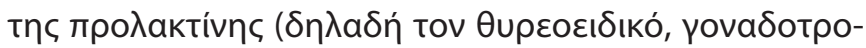

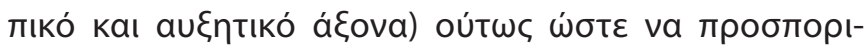

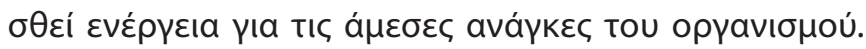

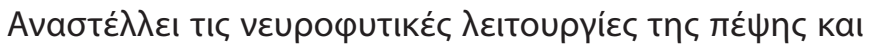

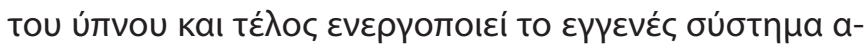
vooíac. ${ }^{14}$

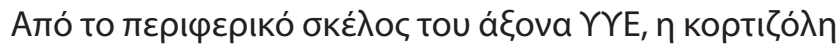

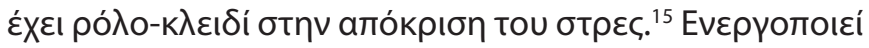

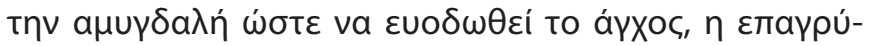

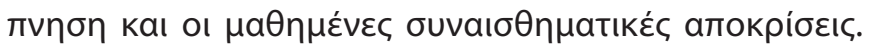

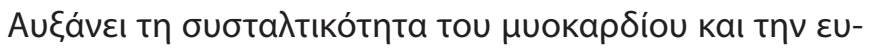

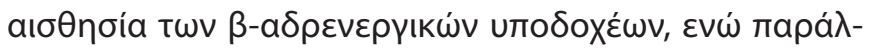

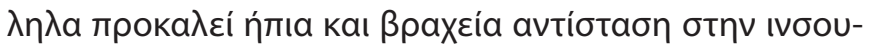

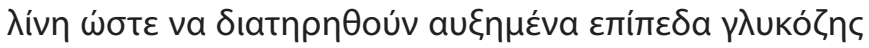

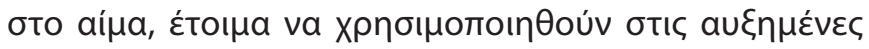

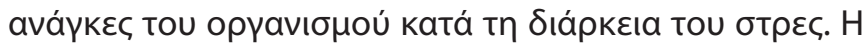

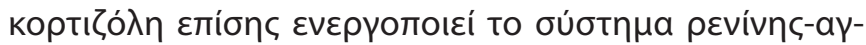

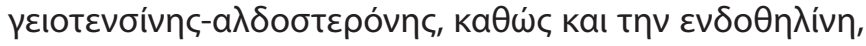

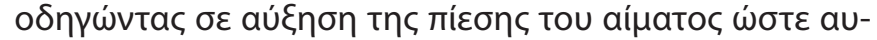

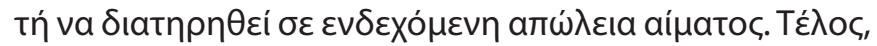

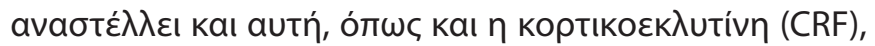

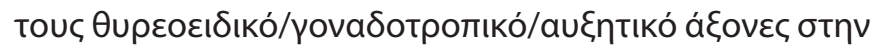

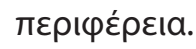

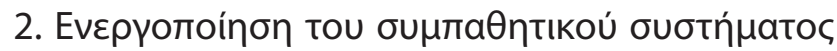

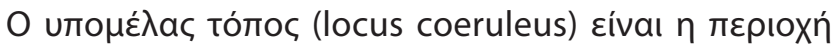

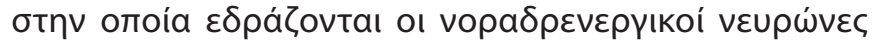

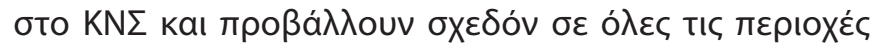

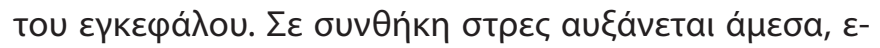

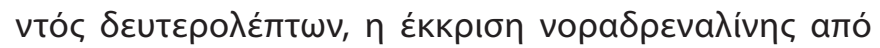

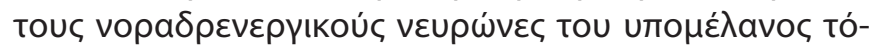

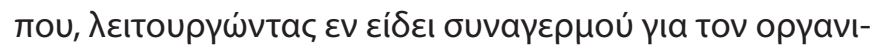

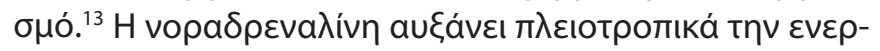

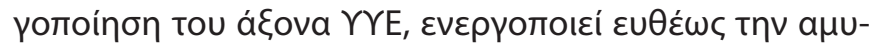

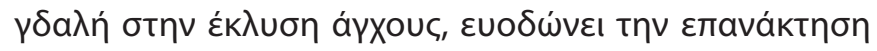

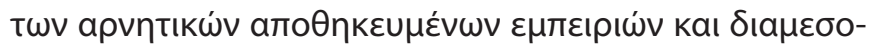

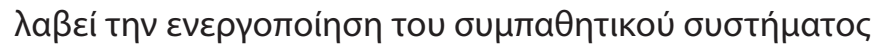

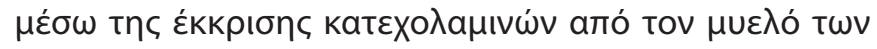

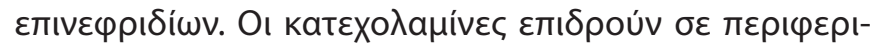

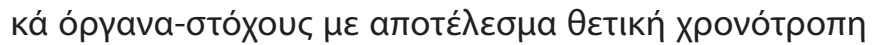

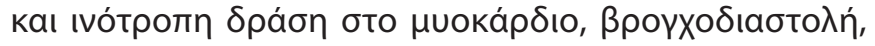




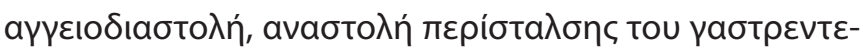

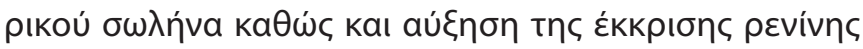

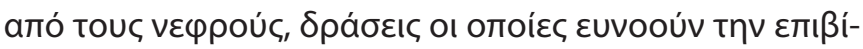

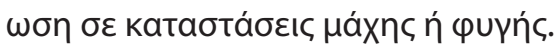

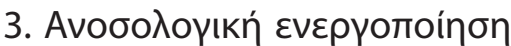

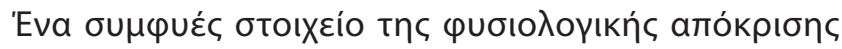

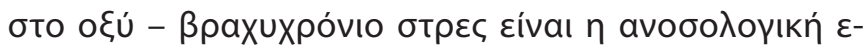

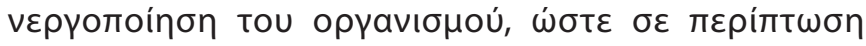

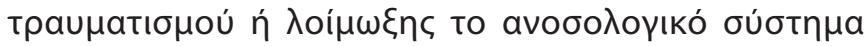

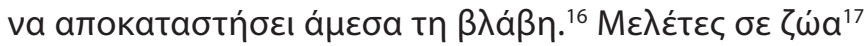

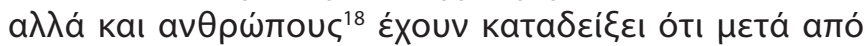

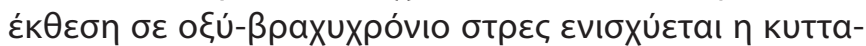

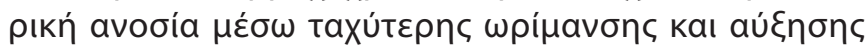

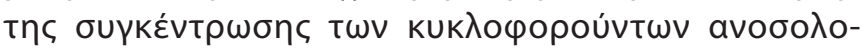

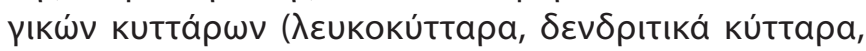

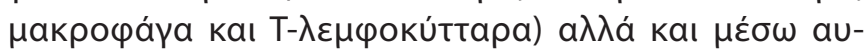

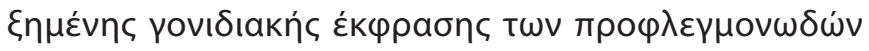

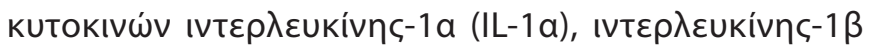

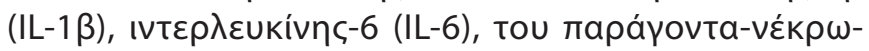

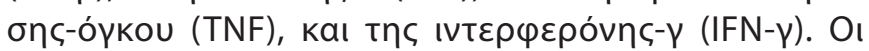

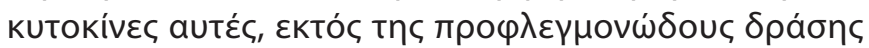

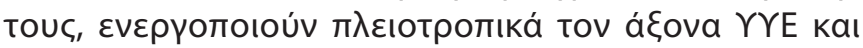

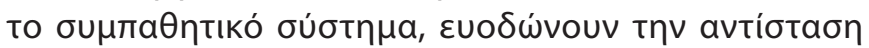

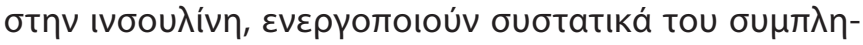

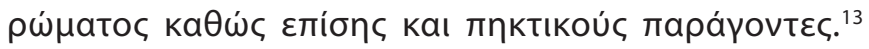

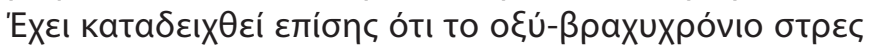

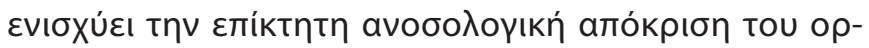

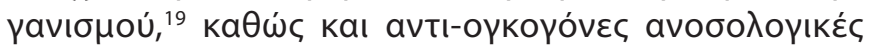

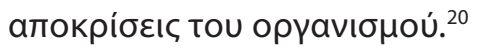

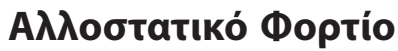

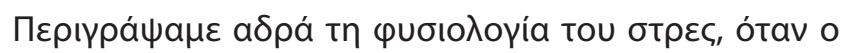

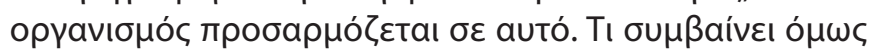

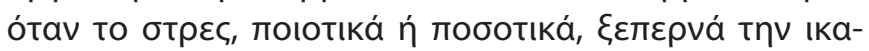

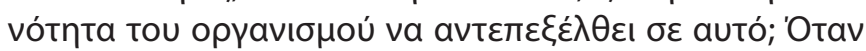

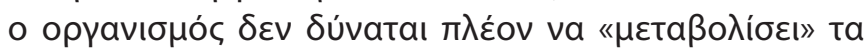

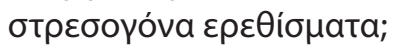

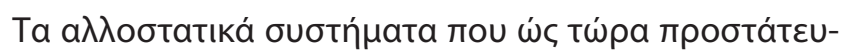

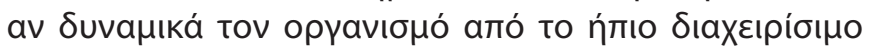

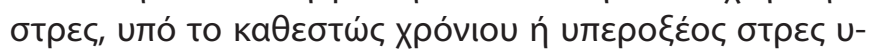

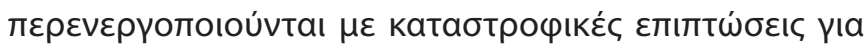

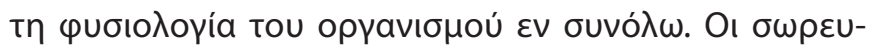

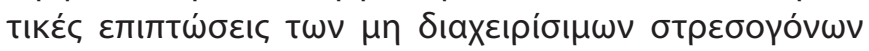

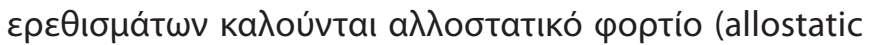

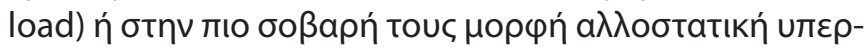

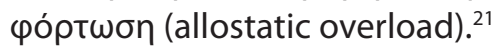

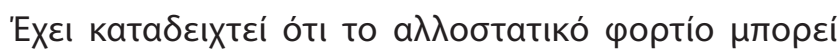

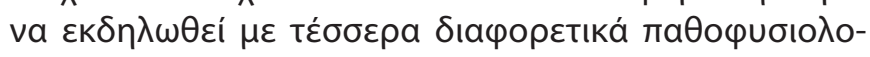

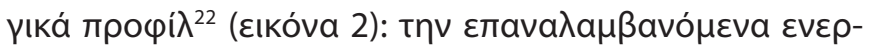

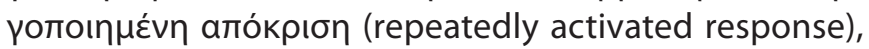

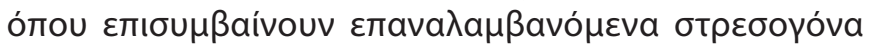

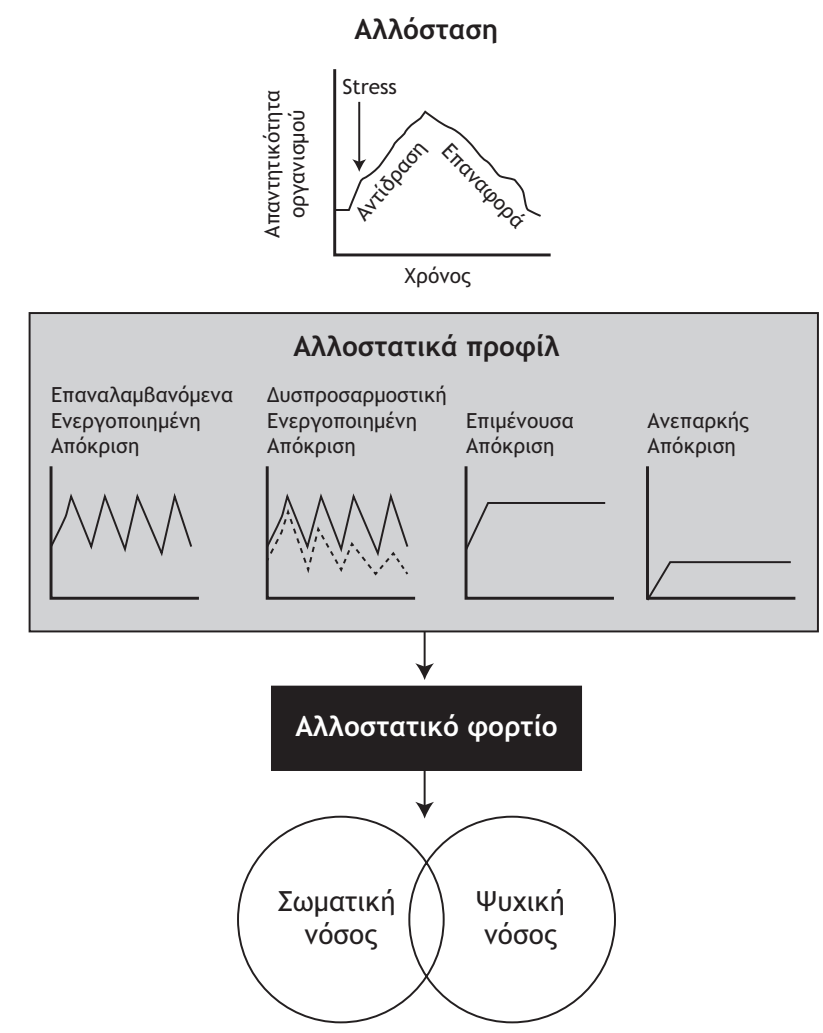

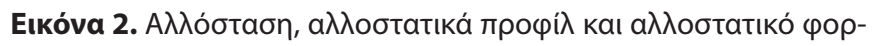

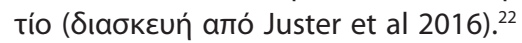

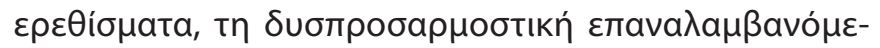

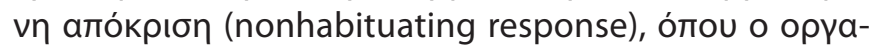

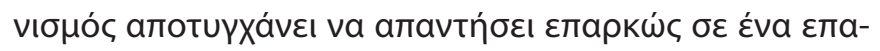

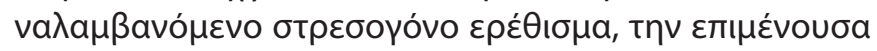

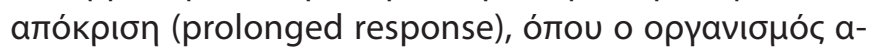

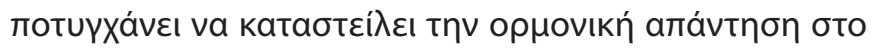

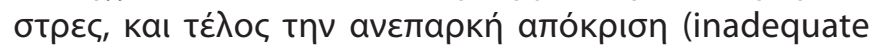

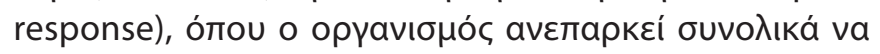

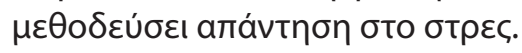

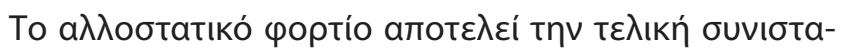

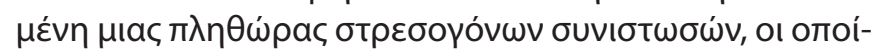

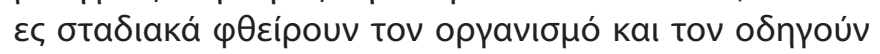

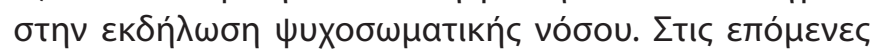

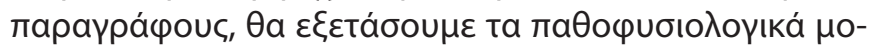

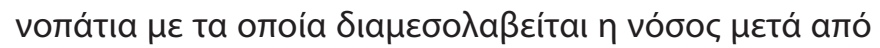

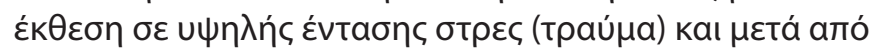

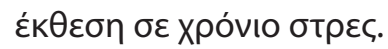

\section{Tpaúra}

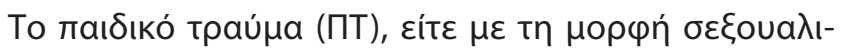

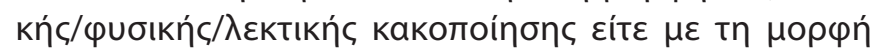

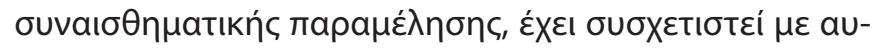

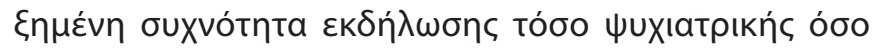

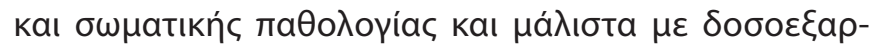

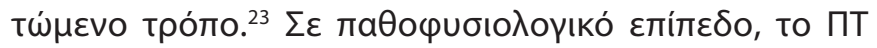




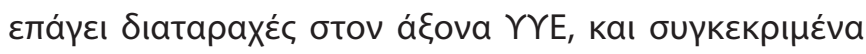

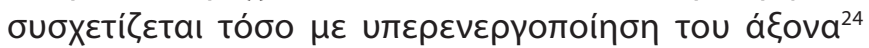

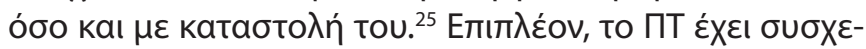

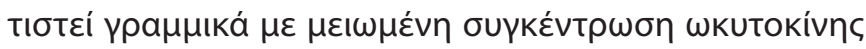

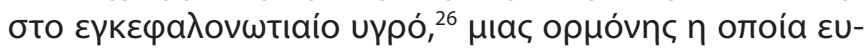

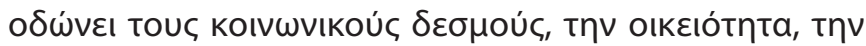

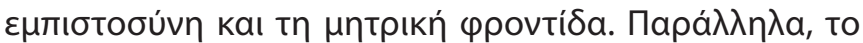

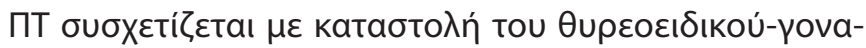

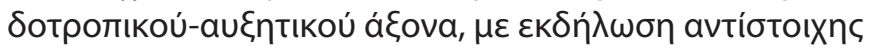

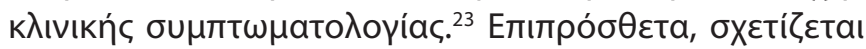

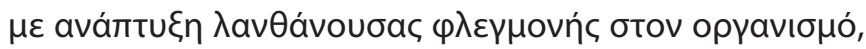

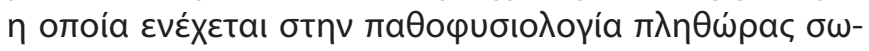

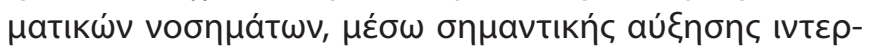

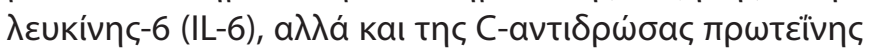

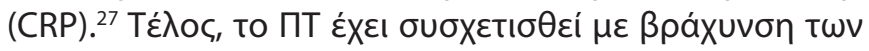

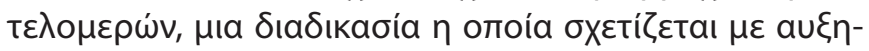

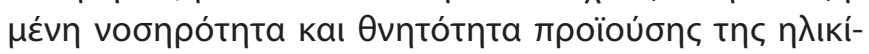
ac.. ${ }^{23}$

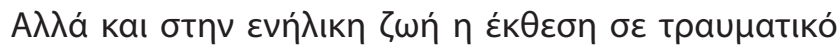

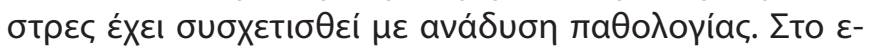

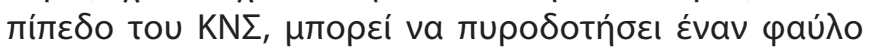

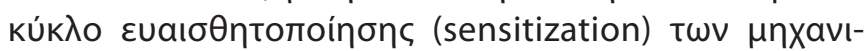

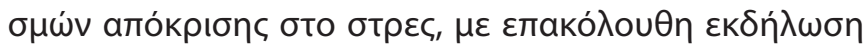

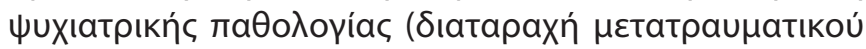

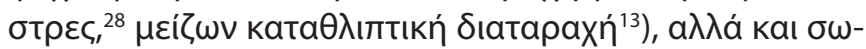

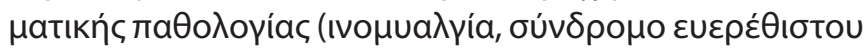

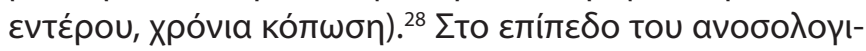

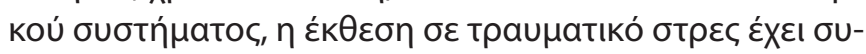

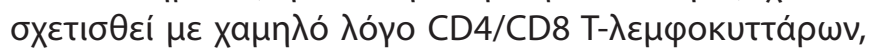

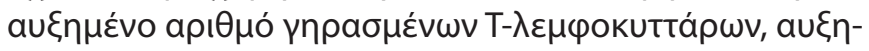

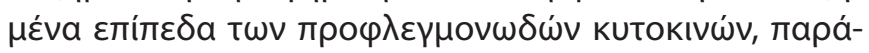

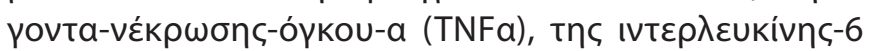

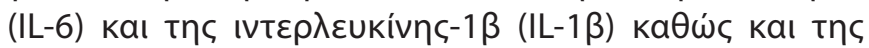

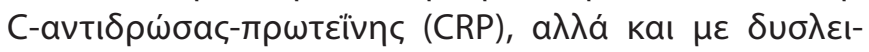

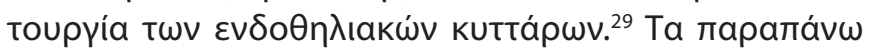

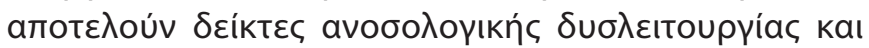

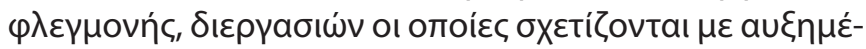

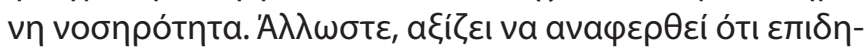

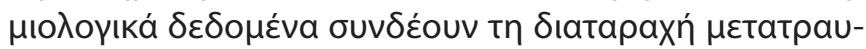

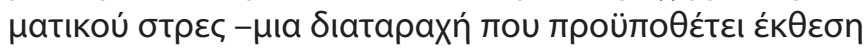

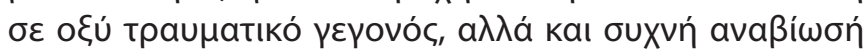

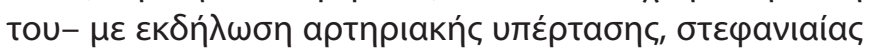

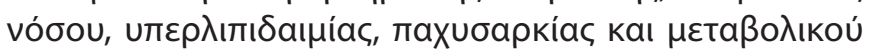

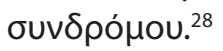

\section{Xpóvio otpec}

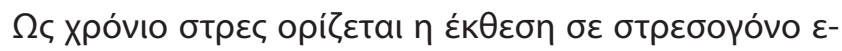

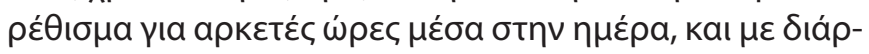

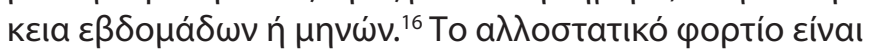

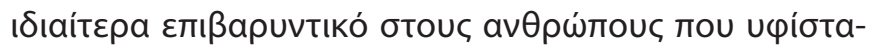

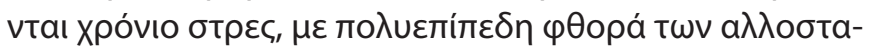

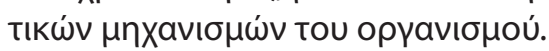

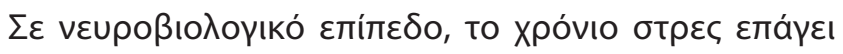

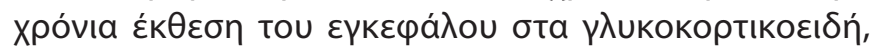

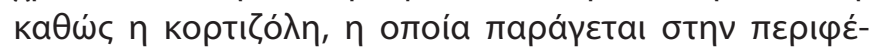

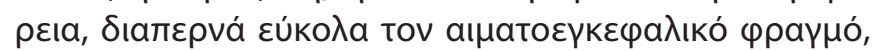

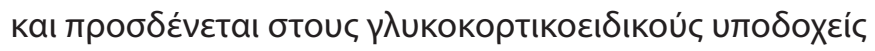

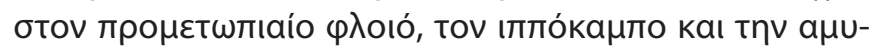

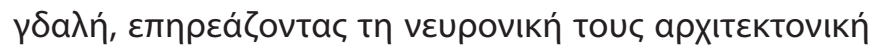

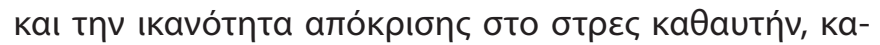

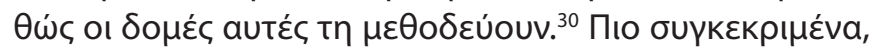

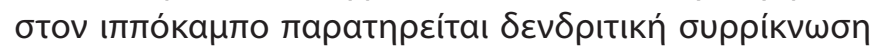

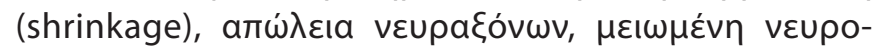

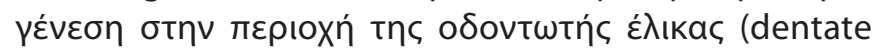

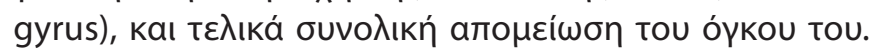

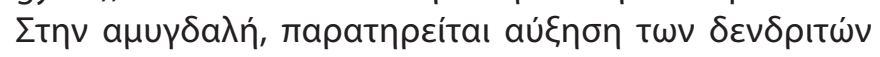

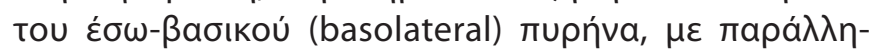

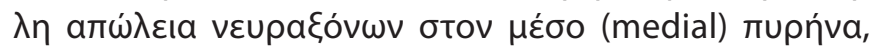

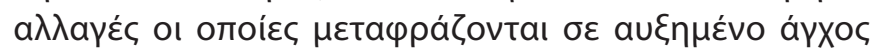

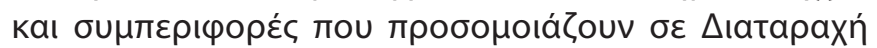

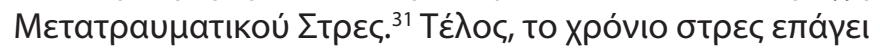

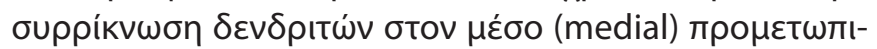

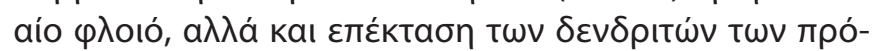

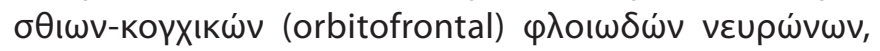

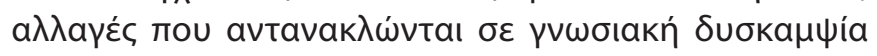

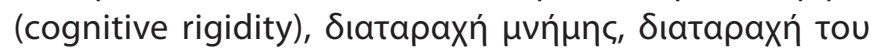

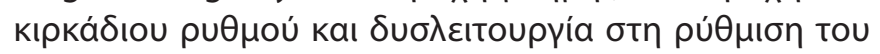

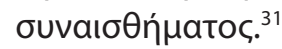

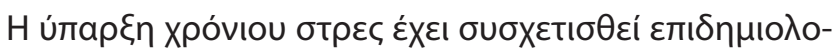

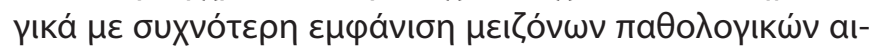

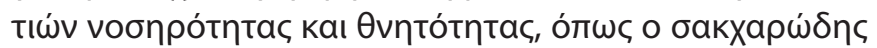

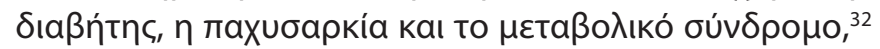

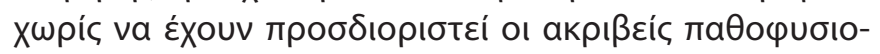

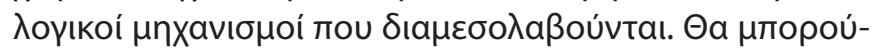

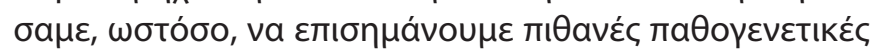
oঠoúc.

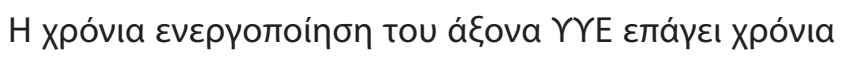

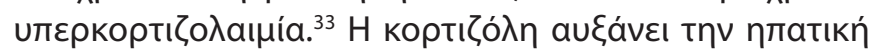

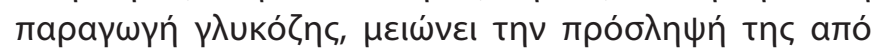

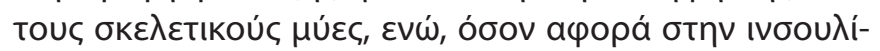

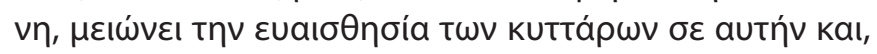

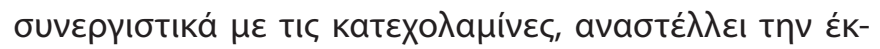

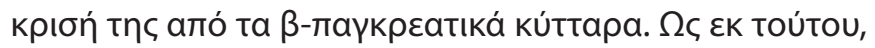

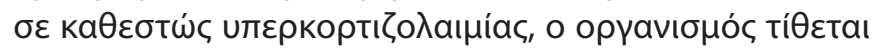

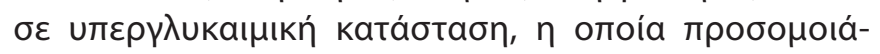

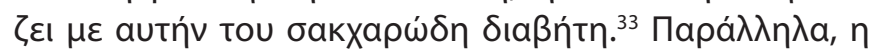

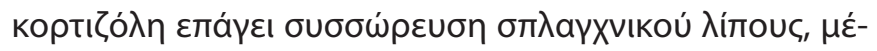

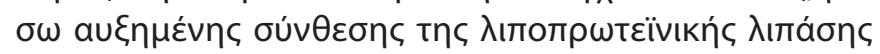

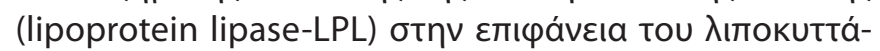

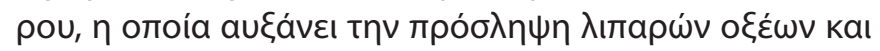

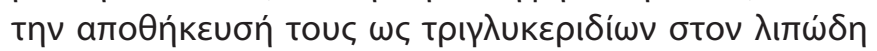

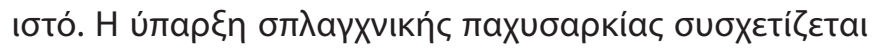

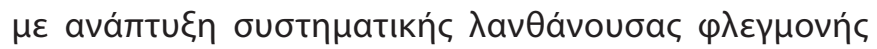

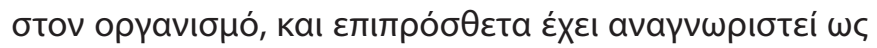




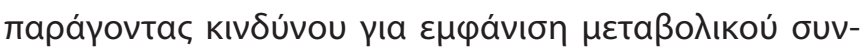
Spópou. ${ }^{34}$

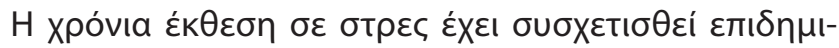

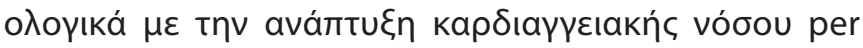

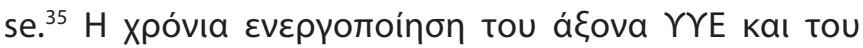

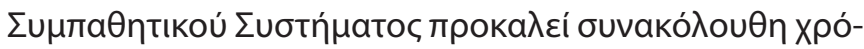

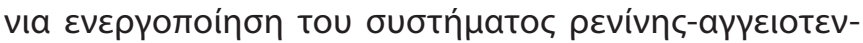

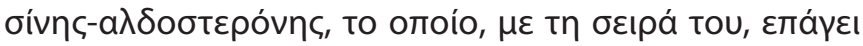

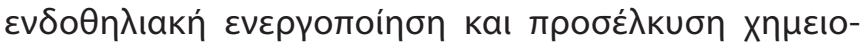

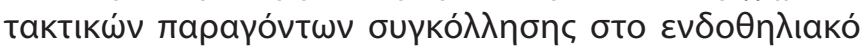

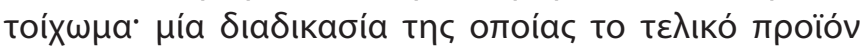

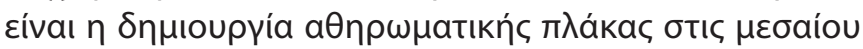

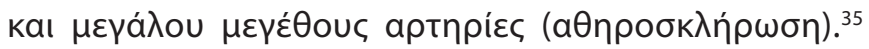

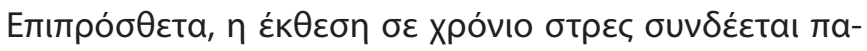

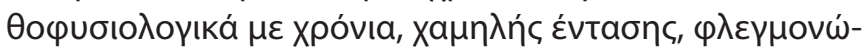

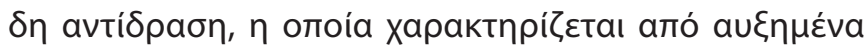

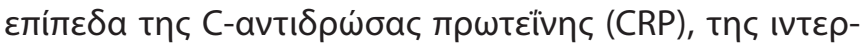

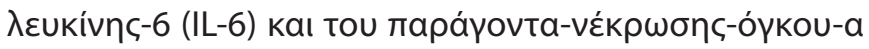

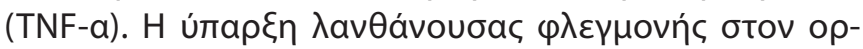

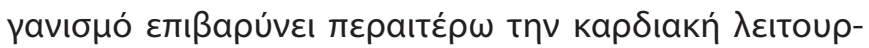

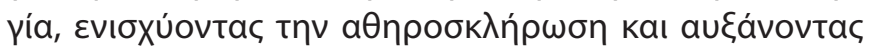

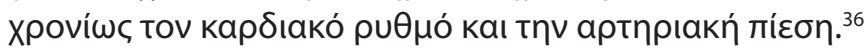

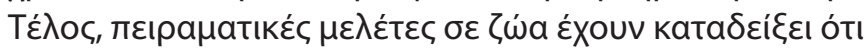

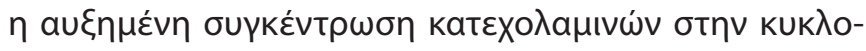

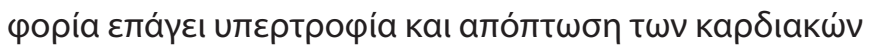

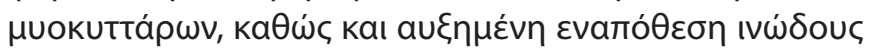

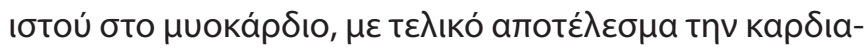

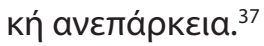

Mía aкó

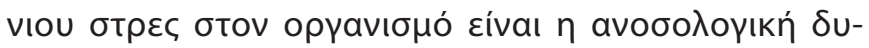

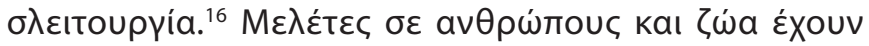

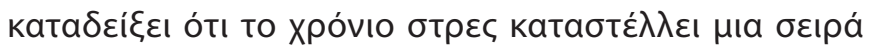

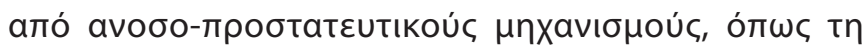

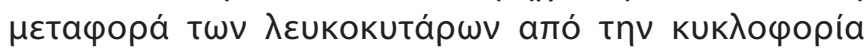

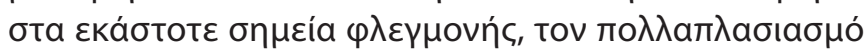

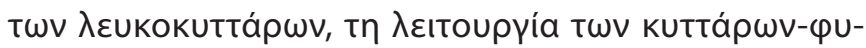

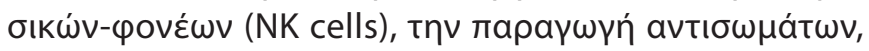

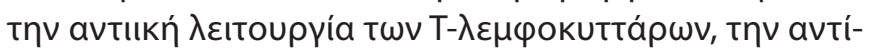

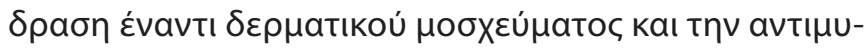

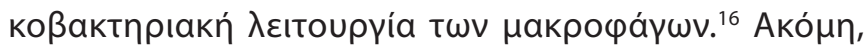

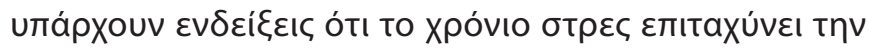

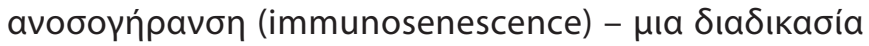

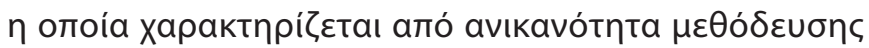

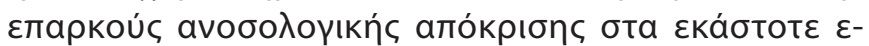

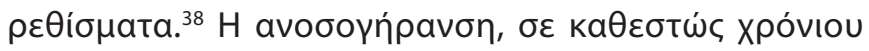

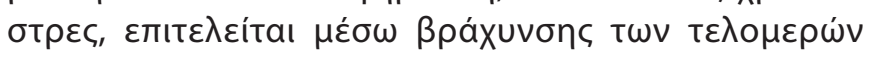

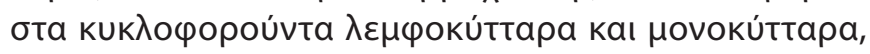

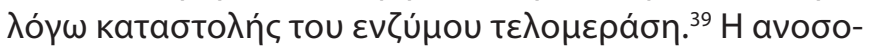

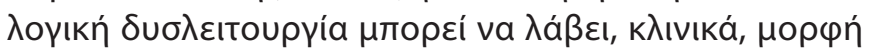

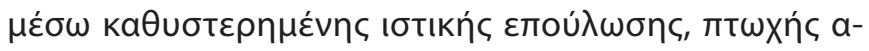

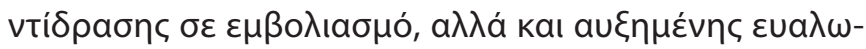

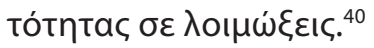

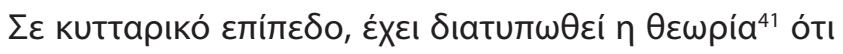

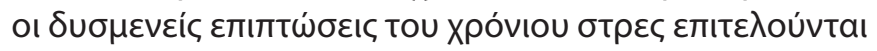

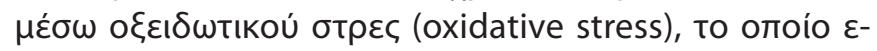

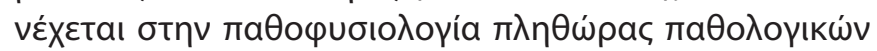

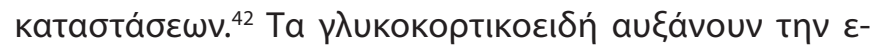

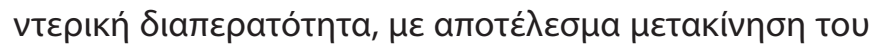

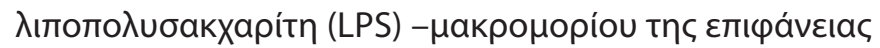

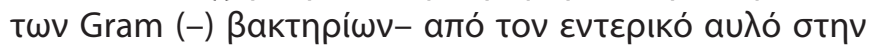

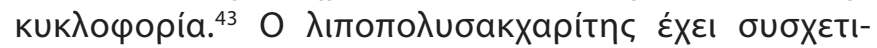

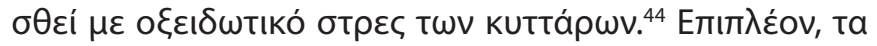

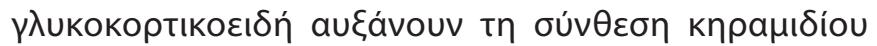

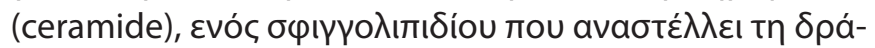

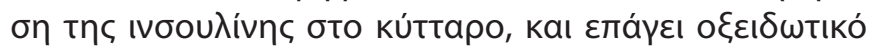

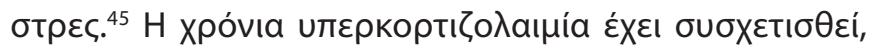

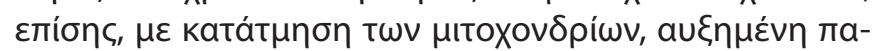

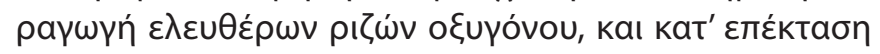

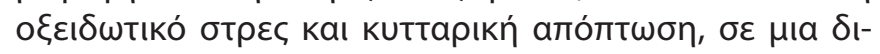

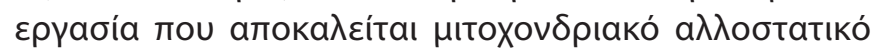
بорті́o. ${ }^{22}$

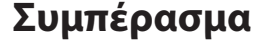

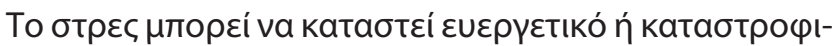

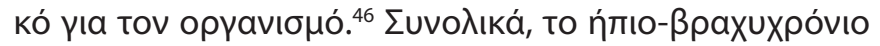

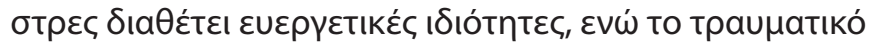

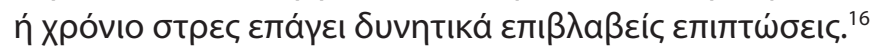

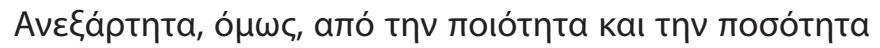

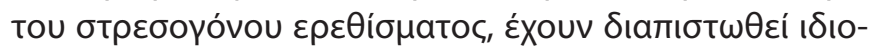

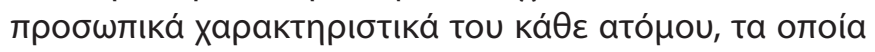

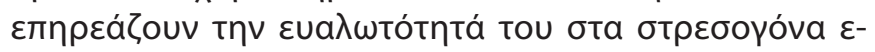

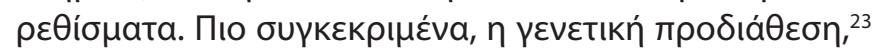

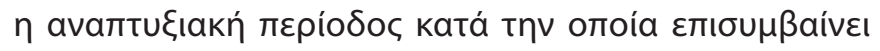

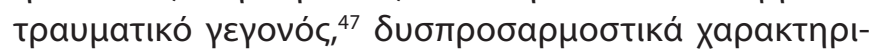

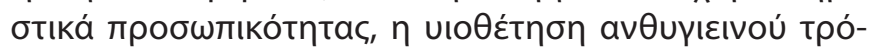

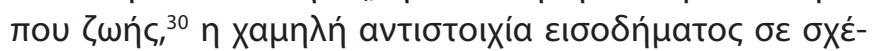

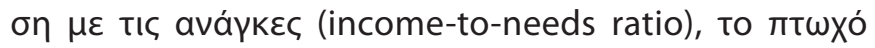

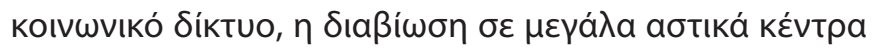

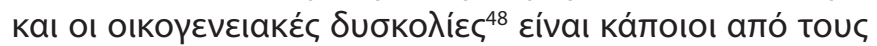

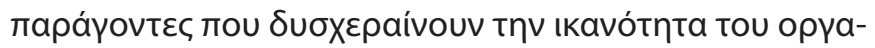

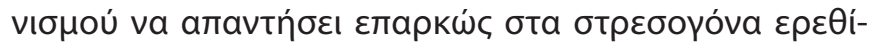

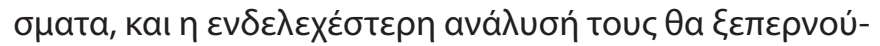

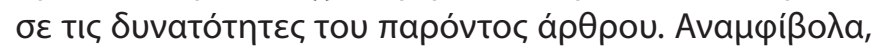

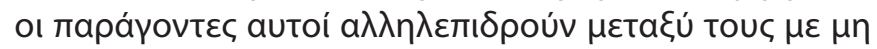

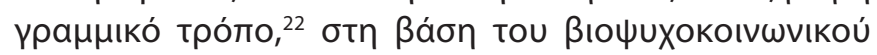

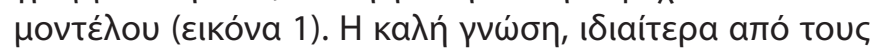

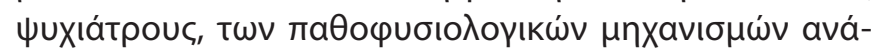

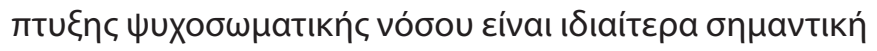

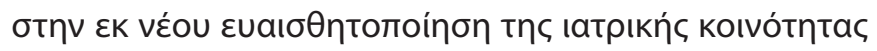

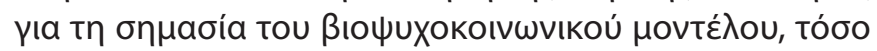

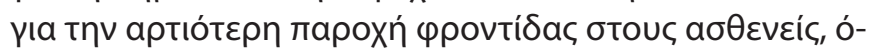

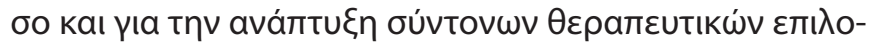

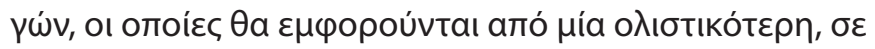

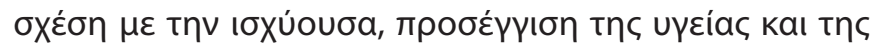
vóoou. 


\section{BıBAıторасрі́a}

1. Engel GL. The need for a new medical model: a challenge for biomedicine. Science 1977, 196:129-136, doi: 10.1126/science.847460

2. Novack DH, Cameron O, Epel E, Ader R, Waldstein SR, Levenstein $S$ et al. Psychosomatic Medicine: The Scientific Foundation of the Biopsychosocial Model. Acad Psychiatry 2007, 31:388-401, doi: 10.1176/ appi.ap.31.5.388

3. Bolton D, Gillett G. The Biopsychosocial Model 40 Years On. In: The Biopsychosocial Model of Health and Disease: New Philosophical and Scientific Developments. Palgrave Pivot, Cham, 2019, doi: 10.1007/9783-030-11899-0_1

4. Christodoulou GN. Psychosomatic Medicine. Plenum Press, New York and London, 1987

5. Christodoulou N. Psychosomatic Medicine in Ancient Greece: An overview. In: Hoyle Leigh (ed) Global Psychosomatic Medicine and Consultation - Liaison Psychiatry: Theory, Research, Education and Practice. Springer, Switzerland, 2019

6. Moussas G, Karkanias A, Christodoulou GN. Psychosomatic Medicine in Modern Greece. In: Hoyle Leigh (ed) Global Psychosomatic Medicine and Consultation - Liaison Psychiatry: Theory, Research, Education and Practice. Springer, Switzerland, 2019

7. Waldstein SR, Neumann SA, Drossman DA, Novack DH. Teaching psychosomatic (biopsychosocial) medicine in United States medical schools: survey findings. Psychosom Med 2001, 63:335-343, doi: 10.1097/00006842-200105000-00001

8. Kusnanto H, Agustian D, Hilmanto D. Biopsychosocial model of illnesses in primary care: A hermeneutic literature review. J Family Med Prim Care 2018, 7:497-500, doi: 10.4103/jfmpc.jfmpc_145_17

9. Danese A, McEwen BS. Adverse childhood experiences, allostasis, allostatic load, and age-related disease. Physiol Behav 2012, 106:29-39, doi: 10.1016/j.physbeh.2011.08.019

10. Sterling P, Eyer J. Allostasis: A new paradigm to explain arousal pathology. In: Fisher S \& Reason J (eds) Handbook of life stress, cognition and health. New York, Wiley, 1988

11. McEwen BS, Seeman T. Protective and damaging effects of mediators of stress: Elaborating and testing the concepts of allostasis and allostatic load. Ann N Y Acad Sci 1999, 896:30-47, doi: 10.1111/j.17496632.1999.tb08103.x

12. Diorio D, Viau V, Meaney MJ. The role of the medial prefrontal cortex (cingulate gyrus) in the regulation of hypothalamic-pituitary-adrenal responses to stress. J Neurosci 1993, 13:3839-3847, doi: 10.1523/ JNEUROSCI.13-09-03839.1993

13. Gold PW. The organization of the stress system and its dysregulation in depressive illness. Mol Psychiatry 2015, 20:32-47, doi: 10.1038/ mp.2014.163

14. Karalis K, Sano H, Redwine J, Listwak S, Wilder RL, Chrousos GP. Autocrine or paracrine inflammatory actions of corticotropin-releasing hormone in vitro. Science 1991, 254:421-423, doi: 10.1126/ science. 1925600

15. Makino S, Gold PW, Schulkin J. Effects of corticosterone on CRH mRNA and content in the bed nucleus of the stria terminalis; comparison with the effects in the central nucleus of the amygdala and the paraventricular nucleus of the hypothalamus. Brain Res 1994, 42:25-28, doi: 10.1016/0006-8993(94)90961-x
16. Dhabhar FS. Effects of stress on immune function: the good, the bad, and the beautiful. Immunol Res 2014, 58:193-210, doi: 10.1007/s12026014-8517-0

17. Viswanathan K, Daugherty C, Dhabhar FS. Stress as an endogenous adjuvant: augmentation of the immunization phase of cell-mediated immunity. Int Immunol 2005, 17:1059-1069, doi: 10.1093/intimm/ dxh286

18. Bosch JA, Berntson GG, Cacioppo JT, Dhabhar FS, MaruchaPT. Acute stress evokes selective mobilization of T cells that differ in chemokine receptor expression: a potential pathway linking immunologic reactivity to cardiovascular disease. Brain Behav Immun 2003, 17:251-259, doi: 10.1016/s0889-1591(03)00054-0

19. Dhabhar FS, McEwen BS. Stress-induced enhancement of antigen-specific cell-mediated immunity. J Immunol 1996, 156:2608-2615, PMID: 8786326

20. Dhabhar FS, Saul AN, Daugherty C, Holmes TH, Bouley DM, Oberyszyn TM. Short-term stress enhances cellular immunity and increases early resistance to squamous cell carcinoma. Brain Behav Immun 2010, 24:127-137, doi: 10.1016/j.bbi.2009.09.004

21. McEwen BS. Physiology and Neurobiology of Stress and Adaptation: Central Role of the Brain. Psysiol Rev 2007, 87:873-904, doi: 10.1152/ physrev.00041.2006

22. Juster RP, Russell JJ, Almeida D, Picard M. Allostatic load and comorbidities: A mitochondrial, epigenetic, and evolutionary perspective. Dev Psychopathol 2016, 28:1117-1146, doi: 10.1017/S0954579416000730

23. Nemeroff CB. Paradise Lost: The Neurobiological and Clinical Consequences of Child Abuse and Neglect. Neuron 2016, 89:892-909, doi: 10.1016/j.neuron.2016.01.019

24. Pesonen AK, Raikkonen K, Feldt K, Heinonen K, Osmond C, Phillips DI, et al. Childhood separation experience predicts HPA axis hormonal responses in late adulthood: a natural experiment of World War II. Psychoneuroendocrinology 2010, 35:758-767, doi: 10.1016/j.psyneuen.2009.10.017

25. Carpenter LL, Tyrka AR, Ross NS, Khoury L, Anderson GM, Price LH. Effect of childhood emotional abuse and age on cortisol responsivity in adulthood. Biol Psychiatry 2009, 66:69-75, doi: 10.1016/j. biopsych.2009.02.030

26. Heim C, Young LJ, Newport DJ, Mletzko T, Miller AH, Nemeroff CB. Lower CSF oxytocin concentrations in women with a history of childhood abuse. Mol Psychiatry 2009, 14:954-958, doi: 10.1038/ mp.2008.112

27. Danese A, Moffitt TE, Pariante CM, Ambler A, Poulton R, Caspi A. Elevated inflammation levels in depressed adults with a history of childhood maltreatment. Arch Gen Psychiatry 2008, 65:409-415, doi: 10.1001/archpsyc.65.4.409

28. McFarlane AC. The long-term costs of traumatic stress: intertwined physical and psychological consequences. World Psychiatry 2010, 9:3-10, doi: 10.1002/j.2051-5545.2010.tb00254.x

29. Wolf EJ, Morrison FG. Traumatic Stress and Accelerated Cellular Aging: From Epigenetics to Cardiometabolic Disease. Curr Psychiatry Rep 2017, 19:75, doi: 10.1007/s11920-017-0823-5

30. Lupien S, Juster RP, Raymond C, Marin MF. The Effects of Chronic Stress on the Human Brain: From Neurotoxicity, to Vulnerability, to Opportunity. Front Neuroendocrinol 2018, 49:91-105, doi: 10.1016/j. yfrne.2018.02.001 
31. McEwenB, Bowles N, Gray J, Hill M, Hunter R, Karatsoreos I et al. Mechanisms of stress in the brain. Nat Neurosci 2015, 18:1353-1363. doi: 10.1038/nn.4086

32. Sinha R Jastreboff $A$. Stress as a common risk factor for obesity and addiction. Biol Psychiatry 2013, 73:827-835, doi: 10.1016/j.biopsych. 2013.01.032

33. Picard M, Juster RP, McEwen BS. Mitochondrial allostatic load puts the 'gluc' back in glucocorticoids. Nat Rev Endocrinol 2014, 10:303-310, doi: 10.1038/nrendo.2014.22

34. Shively C, Register T, Clarkson T. Social Stress, Visceral Obesity, and Coronary Artery Atherosclerosis: Product of a Primate Adaptation. Am J Primatol 2009, 71:742-751, doi: 10.1002/ajp.20706

35. Lagraauw HM, Kuiper J, Bot I. Acute and chronic psychological stress as risk factors for cardiovascular disease: Insights gained from epidemiological, clinical and experimental studies. Brain Behav Immun 2015, 50:18-30, doi: 10.1016/j.bbi.2015.08.007

36. Harrison N, Cooper E, Voon V, Miles K, Critchley HD. Central autonomic network mediates cardiovascular responses to acute inflammation: Relevance to increased cardiovascular risk in depression? Brain Behav Immun 2013, 31:189-196, doi: 10.1016/j.bbi.2013.02.001

37. Liu W, Wang X, Mei Z, Gong J, Gao X, Zhao Y, et al. Chronic stress promotes the progression of pressure overload-induced cardiac dysfunction through inducing more apoptosis and fibrosis. Physiol Res 2015, 64:325-334, PMID: 25536317

38. de Punder K, Heim C, Wadhwa PD, Entringer S. Stress and immunosenescence: The role of telomerase. Psychoneuroendocrinology 2019, 101:87-100, doi: 10.1016/j.psyneuen.2018.10.019

39. Epel E, Blackburn EH, Lin J, Dhabhar FS, Adler NE, Morrow JD, Cawthon RM. Accelerated telomere shortening in response to life stress. Proc Natl Acad Sci 2004, 101:17312-5, doi: 10.1073/pnas.0407162101
40. Glaser R, Kiecolt-Glaser JK. Stress-induced immune dysfunction: implications for health. Nat Rev Immunol 2005, 5:243-251, doi: 10.1038/ nri1571

41. Onyango AN. Cellular Stresses and Stress Responses in the Pathogenesis of Insulin Resistance. Oxid Med Cell Longev 2018, 2018:4321714, doi: $10.1155 / 2018 / 4321714$

42. Pizzino G, Irrera N, Cucinotta M, Pallio G, Mannino F, Arcoraci V, et al. Oxidative Stress: Harms and Benefits for Human Health. Oxid Med Cell Longev 2017, 2017:8416763, doi: 10.1155/2017/8416763

43. Kelly JR, Kennedy PJ, Cryan JF, Dinan TG, Clarke G, Hyland NP. Breaking down the barriers: the gut microbiome, intestinal permeability and stress-related psychiatric disorders. Front Cell Neurosci 2015, 9:392, doi: 10.3389/fncel.2015.00392

44. de Punder K, Pruimboom L. Stress induces endotoxemia and lowgrade inflammation by increasing barrier permeability. Front Immunol 2015, 6:223, doi: 10.3389/fimmu.2015.00223

45. Holland WL, Brozinick JT, Wang LP, Hawkins ED, Sargent KM, Liu Y, et al. Inhibition of ceramide synthesis ameliorates glucocorticoid-, saturated-fat, and obesity-induced insulin resistance. Cell Metabolism 2007, 7:167-179, doi: 10.1016/j.cmet.2007.01.002

46. McEwen BS. In pursuit of resilience: stress, epigenetics, and brain plasticity. Ann N Y Acad Sci 2016, 1373:56-64, doi: 10.1111/nyas.13020

47. Lupien S, McEwen BS, Gunnar M, Heim C. Effects of stress throughout the lifespan on the brain, behavior and cognition. Nat Rev Neurosci 2009, 10:434-445, doi: 10.1038/nrn2639

48. Evans GW. A multimethodological analysis of cumulative risk and allostatic load among rural children. Dev Psychol 2003, 39:924-933, doi: 10.1037/0012-1649.39.5.924 


\title{
Stress and pathophysiological mechanisms for the development of psychosomatic disease
}

\author{
Ioannis Tollos, ${ }^{1}$ Angeliki Theodorakopoulou, ${ }^{2}$ Georgios N. Christodoulou ${ }^{3}$
}

'Second Department of Psychiatry, National and Kapodistrian University of Athens, Athens,

${ }^{2}$ Dromokaiteio Mental Hospital, Athens,

${ }^{3}$ Hellenic Psychiatric Association

${ }^{4}$ Medical School, National and Kapodistrian University of Athens, Athens, Greece

ARTICLE HISTORY: Received 30 March 2020 / Revised 27 July 2020 / Published Online 28 May 2021

\begin{abstract}
According to the biopsychosocial model, disease is the dysfunctional resultant of various fields of human function (biological, psychological, behavioral, socioeconomic). This article deals with the psycho-biological field of Psychosomatic research, namely, the effect of stress on the body. The human organism, in the prospect of evolution, has developed biological mecha-

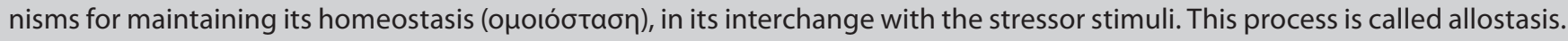
The response to mild - short-term stress induces activation of the hypothalamic - pituitary - adrenal axis, the sympathetic system and the immune system; a process that proves beneficial to the body. On the contrary, exposure to traumatic or chronic stress, with subsequent overactivation of the body's allostatic mechanisms, wears out its homeostatic ability and initiates pathophysiological mechanisms that pave the way for the development of physical and mental illness. The above procedure is called allostatic load or, in its most severe form, allostatic overload. More specifically, the experience of traumatic stress, either in childhood or in adulthood, induces dysregulation of neuroendocrine pathways in the Central Neural System (CNS), as well as immune dysfunction, and is associated with more frequent development of psychiatric and medical pathology, in a dose-dependent way. On the other hand, exposure to chronic repetitive stress induces neurobiological lesions at the level of the CNS, which undermine the body's very ability to respond to stress. At the same time, chronic stress has been associated with increased morbidity from major medical disorders, such as diabetes mellitus, obesity, metabolic syndrome and cardiovascular disease, through a variety of pathophysiological pathways. Finally, chronic stress causes dysfunction of the body's immuno-protective mechanisms, while at a cellular level, it induces oxidative stress and cellular apoptosis. However, regardless of the quantity and quality of the stressor stimuli, it has also emerged that the individual's personal characteristics judge the vulnerability to stress, too. Psychiatrists must insist on the scientific documentation of the biopsychosocial model, so that they can raise awareness amongst the medical community, aiming at a holistic care of the patients.
\end{abstract}

KEYWORDS: Biopsychosocial model, allostasis, allostatic load, trauma, chronic stress, psychosomatic disease.

Corresponding author: Ioannis Tollos, Psychiatric clinic, 2nd Department of Psychiatry, Medical School, National and Kapodistrian University of Athens, "ATTIKON" University Hospital, Rimini 1, GR-124 62 Chaidari, Athens, Greece • e-mail: iw_toll@yahoo.gr 